\title{
High-order interdependencies in the aging brain
}

Marilyn Gatica ${ }^{\mathrm{a}, \mathrm{b}, *}$, Rodrigo Cofré ${ }^{\mathrm{c}_{*}}$, Pedro A.M. Mediano ${ }^{\mathrm{d}}$, Fernando E. Rosas ${ }^{\mathrm{e}, \mathrm{f}, \mathrm{g}}$, Patricio Orio $^{\mathrm{a}, \mathrm{h}}$, Ibai Diez ${ }^{\mathrm{i}, \mathrm{j}, \mathrm{k}}$, Stephan P. Swinnen ${ }^{\mathrm{l}, \mathrm{m}}$, and Jesus M. Cortes ${ }^{\mathrm{n}, \mathrm{o}, \mathrm{p}^{*}}$

${ }^{a}$ Centro Interdisciplinario de Neurociencia de Valparaíso, Universidad de Valparaíso, Pje Harrington 287, 2360103 Valparaíso, Chile.

${ }^{b}$ Biomedical Research Doctorate Program, University of the Basque Country (UPV/EHU), 48940 Leioa, Spain.

${ }^{c}$ CIMFAV-Ingemat, Facultad de Ingeniería, Universidad de Valparaíso, Valparaíso, Chile.

${ }^{d}$ Department of Psychology, University of Cambridge, Cambridge, UK.

e Centre for Psychedelic Research, Department of Brain Science, Imperial College London, London SW7 2DD, UK.

${ }^{f}$ Data Science Institute, Imperial College London, London SW7 2AZ, UK.

${ }^{g}$ Centre for Complexity Science, Imperial College London, London SW7 2AZ, UK.

${ }^{\mathrm{h}}$ Instituto de Neurociencia, Facultad de Ciencias, Universidad de Valparaíso, 2360102 Valparaíso, Chile.

' Department of Radiology, Gordon Center for Medical Imaging, Harvard Medical School, Massachusetts General Hospital, Boston, MA, USA.

${ }^{j}$ Neurology Department, Harvard Medical School, Boston, MA, USA.

${ }^{\mathrm{k}}$ Neurotechnology Laboratory, Tecnalia Health Department, Derio, Spain.

' Research Center for Movement Control and Neuroplasticity, Department of Movement Sciences, KU Leuven, Leuven, Belgium.

m Leuven Brain Institute (LBI), KU Leuven, Leuven, Belgium.

${ }^{\mathrm{n}}$ Computational Neuroimaging Lab, Biocruces Health Research Institute, 48903 Barakaldo, Spain.

`IKERBASQUE: The Basque Foundation for Science, 48013 Bilbao, Spain. 
${ }^{\mathrm{p}}$ Department of Cell Biology and Histology, University of the Basque Country, 48940 Leioa, Spain.

*Correspondence to:

Jesus M Cortes Diaz

Computational Neuroimaging Lab.

Biocruces Health Research Institute.

Cruces University Hospital,

Plaza de Cruces S/N, E-48903,

Barakaldo, Bizkaia, Spain

Tel +34 946006000 (ext. 5199)

E-mail: jesus.m.cortes@gmail.com

Keywords: Redundancy; Synergy; Functional Magnetic Resonance Imaging; Resting state; Aging; High-order interactions

PACS: 87.19.la, 89.70.+c, 89.90.+n 
Abstract

Background: Brain interdependencies can be studied from either a structural/anatomical perspective ("structural connectivity"-SC) or by considering statistical interdependencies ("functional connectivity"-FC). Interestingly, while SC is by definition pairwise (whitematter fibers project from one region to another), FC is not. However, most FC analyses only focus on pairwise statistics and they neglect higher-order interactions. A promising tool to study high-order interdependencies is the recently proposed O-Information, which can quantify the intrinsic statistical synergy and the redundancy in groups of three or more interacting variables.

Methods: We analyzed functional magnetic resonance imaging (fMRI) data obtained at rest from 164 healthy subjects with ages ranging in 10 to 80 years and used O-Information to investigate how high-order statistical interdependencies are affected by age.

Results: Older participants (from 60 to 80 years old) exhibited a higher predominance of redundant dependencies as compared to younger participants, an effect that seems to be pervasive as it is evident for all orders of interaction. In addition, while there is strong heterogeneity across brain regions, we found a 'redundancy core' constituted by the prefrontal and motor cortices in which redundancy was evident at all the interaction orders studied.

Discussion: High-order interdependencies in $\mathrm{fMRI}$ data reveals a dominant redundancy in functions such as working memory, executive and motor functions. Our methodology can be used for a broad range of applications, and the corresponding code is freely available. 
Impact statement

Past research has showcased multiple changes to the brain's structural and functional properties caused by ageing. Here we expand prior work through recent advancements in multivariate information theory, which provide richer and more theoretically principled analyses than existing alternatives. We show that the brains of older participants contain more redundant information at multiple spatial scales -- i.e. activation in different brain regions is less diverse, compared to younger participants -- and identify a "redundancy core" constituted by prefrontal and motor cortices, which might explained impaired performance in the old population in functions such as working memory and executive control. 
List of acronyms:

fMRI: functional magnetic resonance imaging

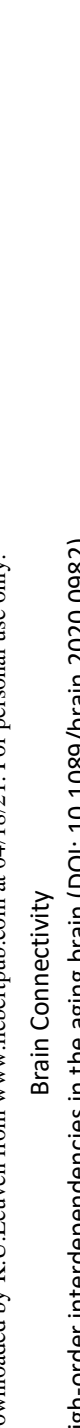

SC: structural connectivity

FC: functional connectivity

MFG: middle frontal gyrus

BHA: brain hierarchical atlas

DMN: default mode network

FDR: false discovery rate

TC: total correlation

DTC: dual total correlation 


\section{Introduction}

A better understanding of the anatomical and functional correlates of aging in the brain will have significant scientific, medical and social implications, particularly as predictions suggest that the elderly population might double worldwide from 2015 to 2050 (World Health Organization, 2018). Aging causes systemic decline across multiple scales, affecting biological, cognitive and psychosocial outcomes. For example, aging disrupts circadian and sleep cycles, causing a loss in sleep quality (Kondratova et al., 2012) that can impair different domains of cognitive performance, such as information processing speeds, working memory, executive functions and reasoning (Grady, et al., 2012): In turn, this may exacerbate mental health conditions like anxiety and depression (Knight, et al., 2015).

Age-related cognitive decline occurs in parallel with well-established variations in brain morphology. During an individual's lifespan, the total brain volume increases from childhood to adolescence by about $25 \%$ on average, remaining constant over the following three decades and, ultimately, decaying towards childhood sizes at later ages (Courchesne, et al., 2000). Notably, the atrophy in aged brains is not uniform but rather, some anatomical regions are more strongly affected than others, with the hippocampus (West, et al., 1993) and the prefrontal cortex (Sullivan, et al., 2007) structures known to be targeted for age-related atrophy.

Resting state functional magnetic resonance imaging or functional MRI (fMRI) has shown that aging affects functional connectivity (FC) in extended brain networks, specifically altering anterior and posterior regions like the superior and middle frontal gyrus (MFG), posterior cingulate, middle temporal gyrus and the superior parietal region (Damoiseaux, et al., 2008; Andrews-Hanna, et al., 2007). Other studies have shown that aging may also alter the FC between several regions, possibly indicating compensation or pathological activation (Betzel, et al., ,2014; Chan, et al., 2014; Onoda, et al., 2012; Solesio-Jofre, et al., 2014).

An important limitation of such FC studies is that their analysis is restricted to pairwise FC, ignoring possible higher order effects. High-order interactions allow us to distinguish redundancy and synergy dominated interactions that play key roles in neural dynamics (Friston, et al., 2003; Schneidman, et al., 2003; Erramuzpe, et al., 2015; Stramaglia, et al., 
2016; Ince, et al., 2017a; Ince, et al., 2017b; Battiston, et al., 2020; Stramaglia, et al., 2021). An initial study of high-order interactions and aging showed significant changes in the synergies and redundancies of triple interactions over the individual's lifespan (Camino-Pontes, et al., 2018), as well as a redundant role of the default mode network (DMN). However, the effects of aging on interactions beyond triple relationships remain largely unexplored to the best of our knowledge.

Here, we built on our previous studies (Camino-Pontes, et al., 2018) to examine the effects of aging on higher order interactions in the human brain, paying special attention to the interdependencies between four or more brain regions. We employed the recently proposed O-Information (Rosas, et al., 2019), which can be considered a revised version of the measure of neural complexity proposed previously in the context of Partial Information Decomposition (Tononi, et al., 1994; Williams, et al., 2010). More specifically, O-Information captures the balance between redundancies and synergies in arbitrary sets of variables, thereby extending the properties of the interaction information of three variables to larger sets (McGill, 1954). Redundancy is understood here as an extension of the conventional notion of the correlation between more than two variables, in which each variable has a "copy" of some common information shared with other variables (Rosas, et al., 2018). An example of extreme redundancy is full synchronization, where the status of one signal allows one to predict the status of any other. By contrast, synergy corresponds to statistical relationships that regulate the whole but not the parts (Rosas, et al., 2019; Timme, et al., 2014; Wibral, et al., 2017). Synergy allows local independency and global cohesion to coexist, a condition that has been recently found to be instrumental for higher order brain functions (Luppi, et. al, 2020a; Luppi et. al, 2020b), while redundancy including highly synchronized situations like deep sleep stages or epileptic seizures - would make brains less well-suited to this (Tononi, et al., 1994; Tononi, et al., 1998).

To investigate how the higher order informational organization of the brain changes with age, we studied synergic and redundant interactions of different orders from fMRI data obtained in the resting state from a cohort of 164 healthy volunteers aged 10 to 80 years old. This approach is novel for two reasons. From a methodological perspective, we present the first application of higher order statistical interdependencies in aging, beyond 
triplet interactions, developing a formalism to apply the metrics previously presented to fMRI data (Rosas, et al., 2019). In addition, we investigate how aging alters the higher order interdependencies among different brain regions, as inferred directly from fMRI data. In this way, we obtained novel insights into brain aging, whereby the interdependencies in older participants appear to be more redundancy-dominated than those in younger participants, for all interaction orders. When studying how these effects are distributed topographically, we found a "redundancy core" that was composed of brain regions that play key roles in the most redundancy-dominated arrangements for all interaction orders.

\section{Materials and Methods}

\section{Participants}

A cohort of healthy participants $(P=164)$ were recruited in this study, aged from 10 to 80 years (mean age 44.35 years, SD 22.14 years). Informed consent was obtained from all the subjects before they were enrolled onto the study, in accordance with the local ethics committee guidelines for clinical research and the Helsinki Declaration.

We grouped the participants into four age groups li for $i \in\{1, \ldots, 4\}$, more specifically: I1 consists of 30 subjects aged $10-20$ years old; 12 , 46 subjects $20-40$ years old; 13,29 subjects 40-60 years old; and 1459 subjects $60-80$ years.

\section{Image acquisition and analyses}

MRI images were acquired with a Siemens 3T MAGNETOM Trio MRI scanner using a 12channel matrix head coil. The anatomic data was acquired as a high-resolution T1 image with 3D magnetization prepared rapid acquisition gradient echo (MP-RAGE): repetition time $(\mathrm{RT})=2300 \mathrm{~ms}$, echo time $(\mathrm{ET})=2.98 \mathrm{~ms}$, voxel size $=1 \times 1 \times 1.1 \mathrm{~mm}^{3}$ slice thickness $=1.1 \mathrm{~mm}$, field of view $=256 \times 240 \mathrm{~mm}^{2}$, with 160 contiguous sagittal slices covering the entire brain and brainstem. Resting state functional data was acquired with a gradient echo-planar imaging sequence over a 10 min session using the following parameters: 200 whole brain volumes with TR/TE $=3000 / 30 \mathrm{~ms}$, flip angle $=90$, interslice gap $=0.28 \mathrm{~mm}$, voxel size $=2.5 \times 3 \times 2.5 \mathrm{~mm}^{3}, 80 \times 80$ matrix, slice thickness $=2.8 \mathrm{~mm}, 50$ oblique axial slices interleaved in descending order. 
Image preprocessing was performed following a similar procedure to that used in Ref. (Camino-Pontes, et al., 2018) and subsequently, each subject was represented by 2,514 time series of the fMRI signal (Fig. 1). These series were grouped into 20 new time series by spatial-averaging, corresponding to the $M=20$ module partition of the Brain Hierarchical Atlas (BHA) (Diez, et al., 2015), the partition that maximizes functional modularity, structural modularity, and the similarity between the functional and structural modules (for more details see Diez, et al., 2015).

\section{Multivariate information-theoretic metrics}

O-Information (Rosas, et al., 2019) (shorthand for information about organizational structure) is an attempt to operationalize the original desiderata (Tononi, et al., 1994) while overcoming some of the shortcomings of this original proposal (see e.g. Van Putten, et al., 2001; van Walsum, et al., 2003). Denoted by $\Omega\left(X^{n}\right)$, O-Information is a real-value measure whose sign serves to discriminate between redundant and synergistic components: $\Omega\left(X^{n}\right)>0$ corresponds to redundancy-dominated interdependencies; while $\Omega\left(X^{n}\right)<0$ characterizes synergy-dominated ones. When applied over groups of three variables, $\Omega\left(X^{3}\right)$ is equal to the McGill's interaction information (McGill, 1954), although their values differ for larger systems. Importantly, $\Omega\left(X^{n}\right)$ but not the interaction information retains the ability to discriminate between redundancy- and synergydominated systems when $n>3$ (Williams, et al., 2010).

In addition to O-Information, we also assessed the S- information (Rosas, et al., 2019) denoted by $\Sigma\left(X^{n}\right)$, to quantify the strength of multivariate correlations. S-Information is a natural complement to $\mathrm{O}$-Information as it quantifies the overall strength of the interdependencies, while the latter determines whether they are synergistic or redundant in nature. Furthermore, following Ref. (Camino-Pontes, et al., 2018), we take the positive and negative parts of O-Information as heuristic metrics of redundancy and synergy.

Below, we provide details regarding the main information-theoretic metrics used in this study. (Rosas, et al., 2019). The O-Information of a set of $n$ random variables $X_{n}=$ $\left(X_{1}, \ldots, X_{n}\right)$ is calculated as

$$
\Omega\left(X_{n}\right)=T C\left(X_{n}\right)-\operatorname{DTC}\left(X_{n}\right)
$$


while their S-Information is

$$
\Sigma\left(X_{n}\right)=\operatorname{TC}\left(X_{n}\right)+\operatorname{DTC}\left(X_{n}\right)
$$

In these formulae, TC and DTC correspond to the total correlation (Watanabe, 2010) and dual total correlation (Han, 1978), respectively, given by:

$$
\begin{gathered}
\operatorname{TC}\left(X_{n}\right)=\sum_{i=1}^{n} H\left(X_{i}\right)-H\left(X_{n}\right), \\
\operatorname{DTC}\left(X_{n}\right)=H\left(X_{n}\right)-\sum_{i=1}^{n} H\left(X_{i} \mid X_{-i}^{n}\right)
\end{gathered}
$$

Here, $H(\cdot)$ represent the Shannon entropy, and $X_{-i}^{n}$ represent the vector of $n-1$ variables composed by all minus $X_{i}$ i.e., $\left(X_{1}, \ldots, X_{i-1}, X_{i+1}, \ldots, X_{n}\right)$. Both the TC and DTC are non-negative generalizations of mutual information, whereby they are zero if and only if all variables $X_{1}, \ldots, X_{n}$ are statistically independent of one another.

All these quantities were computed for each subject separately using Gaussian Copulas ${ }^{1}$ (Ince, et al., 2017b). The O-Information and S-Information estimated via the data of the $k$-th subject is denoted as $\Omega_{(k)}$ and $\Sigma_{(k)}$, respectively. All these quantities are always computed using natural logarithms.

Interaction order, redundancy and synergy

To estimate the O-Information and S-Information of different brain modules, we calculated the following quantities for each participant:

$$
\Omega_{n}^{m}(k)=\frac{1}{Z_{n}} \sum_{i_{1}} \ldots \sum_{i_{n-1}} \Omega_{(k)}\left(X_{m}, X_{i_{1}}, \ldots, X_{i_{n-1}}\right)
$$

\footnotetext{
${ }^{1}$ This approach exploits the fact that the Mutual Information does not depend on the marginal distributions and therefore, the different quantities can be conveniently transformed into Gaussian random variables for which efficient parametric estimates of high-order interactions exist (Ince, et al., 2017b).
} 


$$
\Sigma_{n}^{m}(k)=\frac{1}{Z_{n}} \sum_{i_{1}} \ldots \sum_{i_{n-1}} \Sigma_{(k)}\left(X_{m}, X_{i_{1}}, \ldots, X_{i_{n-1}}\right),
$$

Above, $k$ is the participant index and $m$ is the module index, $n$ interaction order, and

$$
Z_{n} \equiv\left(\begin{array}{c}
M-1 \\
n-1
\end{array}\right)
$$

is the total number of subsets of size $n-1$ in an atlas of $M$ modules (in this paper we use $M=20$ ). Please note that the summations in Eqs. (3) and (4) are intended to include all the $Z_{n} n$-plets that includes $X_{m}$, once each. Finally, the grand average O-Information and S-Information of order $n$ are calculated as

$$
\begin{aligned}
& \Omega_{n}(k)=\frac{1}{M} \sum_{m=1}^{M} \Omega_{n}^{m}(k), \\
& \Sigma_{n}(k)=\frac{1}{M} \sum_{m=1}^{M} \Sigma_{n}^{m}(k) .
\end{aligned}
$$

We then split the values of O-Information on positive and negative values using

$$
\Omega^{+}=\max \{\Omega, 0\} \quad ; \quad \Omega^{-}=-\min \{\Omega, 0\},
$$

so that $\Omega=\Omega^{+}-\Omega^{-}$. Using these quantities, we estimated the following proxies for the redundancy and synergy per subject $k$, module $m$ and interaction order $n$ :

$$
R_{n}^{m}(k)=\frac{1}{N_{n, m}^{+}} \sum_{i_{1}} \ldots \sum_{i_{n-1}} \Omega_{(k)}^{+}\left(X_{m}, X_{i_{1}}, \ldots, X_{i_{n-1}}\right)
$$




$$
S_{n}^{m}(k)=\frac{1}{N_{n, m}^{-}} \sum_{i_{1}} \ldots \sum_{i_{n-1}} \Omega_{(k)}^{-}\left(X_{m}, X_{i_{1}}, \ldots, X_{i_{n-1}}\right)
$$

where $N_{n, m}^{+}$and $N_{n, m}^{-}$represent the number of $n$-plets with positive and negative OInformation values, respectively. Finally, the average of these quantities over all subjects and modules is calculated as

$$
\begin{aligned}
& R_{n}(k)=\frac{1}{M} \sum_{m=1}^{M} R_{n}^{m}(k), \\
& S_{n}(k)=\frac{1}{M} \sum_{m=1}^{M} S_{n}^{m}(k) .
\end{aligned}
$$

\section{Statistical analyses}

In this study, we compared the group of older participants (14) with the combination of the three other groups $(11,12,13)$. Different information-based measures were compared using a non-parametric statistical Wilcoxon rank sum test. When appropriate, significance levels for hypothesis testing were corrected for multiple comparisons by controlling the false discovery rate (FDR) following a standard Benjamini-Hochberg procedure (Benjamini, et al., 1995).

\section{Results}

We analysed resting-state fMRI data from 164 healthy subjects, measuring the SInformation and O-Information in order to quantify high-order interactions between brain areas in terms of their redundancy and synergy. A scheme of the approach adopted is shown in Fig. 1. Participants were divided into four age groups (I1-14) as described in the 
Materials and Methods.

In this study, we first measured the S-Information and O-Information per age group, and the interaction order. Significant differences were evident in both the S-Information and O-Information between the old group and the younger groups after correcting with the FDR for multiple comparisons (Fig. 2). The increase in S-Information with age implies an increase of interdependencies between the various brain regions. Interestingly, the increase shown in the older population was significant at all orders, suggesting a widespread effect. The increase in the O-Information detected suggests that the correlations in the older population are generally redundancy-dominated, becoming stronger at higher orders. We split the interactions according to the sign of the OInformation, with the positive values representing a measure of redundancy, and the negative ones, of synergy. Analyzing the O-Information, we identified different patterns of redundancy and synergy associated with increasing interaction order (see Fig. 3). While synergy exhibited a bell-shaped curve, redundancy increased linearly with the interaction order. Importantly, the redundancy values were much larger than the synergistic ones and moreover, the redundancy of 14 showed significant differences from that in the group formed by I1, 12 and 13 for all interaction orders. In terms of synergy, while for some interaction orders the 14 group exhibited significant differences from the rest of the population, these differences did not survive a multiple comparison correction.

We quantified the redundancy across brain modules by taking into account all the redundant interactions in which a given module participates. When studying redundancy across the distinct brain areas, modules 1-3, 5, 13-15, 18- 20 exhibited significant differences for all interaction orders while the others only exhibited significant differences for large interaction orders (Fig. 4). This suggests the existence of a redundancy core, as confirmed by later analyses. By contrast, the pattern of synergy across brain areas is very heterogeneous, with only modules 15, 17, 18 and 19 showing significant differences.

To confirm the existence of a redundancy core, we studied the extreme O-Information values for various interaction orders. We found that modules 2, 5 and 13 participate in the most redundancy-dominated $n$-plets for all orders (Fig. 5), suggesting that they might constitute the basis of such a core. While a complete anatomical description of these 
modules is available in Ref. (Diez, et al.,2015; Diez, et al., 2017), it is important to emphasize that these three modules have the MFG and precentral gyrus (PG) in common, two important structures that have a well-differentiated function. Specifically, while the MFG is part of the prefrontal cortex, which mediates executive control and working memory, the PG is part of the primary motor cortex, both structures known to be affected by aging (Grady, 2012; Grady, 2008; Salat, et al., 2004; Pauwels, et al., 2019).

It is important to note that although the redundant role found in this study for the interaction between the MFG and PG was obtained from participants at rest, it potentially reflects the fact that older participants typically compensate motor behavioral deficits by additional activation of the prefrontal cortex in synchrony with their associated motor areas, while younger participants only activate motor areas to perform the same tasks (Heuninckx, et al., 2008; Santos-Monteiro, et al., 2017; Rueda-Delgado, et al., 2019). Similarly, it has also been shown that older but not young subjects recruit the prefrontal cortex when performing purely movement tasks, such as inhibitory motor control, thereby relying more on cognitive support for the performance of a motor task, while younger subjects perform such tasks in a more automatic manner (i.e.: cognitive penetration into action) (Heuninckx, et al., 2005).

Interestingly, the core existing in younger participants (I1, I2 and I3) seemed to be broader than that in the older population, including modules 9,10 and 16 . The brain structures supporting these modules are the middle and superior frontal gyri, posterior cingulate cortex and the precuneus, all part of the DMN, an important network in the human brain (see Raichle, 2015; Buckner, et al., 2008, and references therein). Although a weaker contribution of the precuneus to the DMN has been proposed (Margulies, et al., 2009), our data confirmed its participation, as indicated elsewhere (Raichle, 2015; Beckmann, et al., 2005).

When comparing these results to those in which a redundant role of the $\mathrm{DMN}$ was shown over the individual's lifespan when $n=3$ (Camino-Pontes, et al., 2018), such a role might breakdown in older participants for higher orders of interaction. This change might reflect network re-adaptation or anticipation of damage, as seen at the onset of other pathologies like the early stage of Alzheimer's disease (Schultz, et al. 2017), after 
concussion (Abbas, et al., 2015) or following multiorgan failure (Jimenez-Marin, et al., 2020).

When the modules that participate in synergistic arrangements are considered, i.e.: in the $n$-plets with smaller O-Information values, those belonging to the redundant core tended not to be involved in very synergistic arrangements, with the exception of modules 9 and 16 that participated in all age groups. These two modules have the posterior cingulate and precuneus in common, which as indicated above are part of the DMN. Based on these results, we might hypothesize a dual redundant-synergistic role of the $\mathrm{DMN}$, and an impairment of this redundant contribution in older participants.

\section{Conclusions}

The present study assesses the higher order redundant and synergistic interactions among brain regions in subjects of various ages. Overall, there was an important increase in the redundant interdependencies in the older population for all interaction orders. In addition, a redundant core of brain modules was observed, the influence of which diminished with age. Together, these findings suggest a change in the balance of differentiation and integration towards more synchronized arrangements.

It is important to note that our analysis goes beyond the traditional brain-network approaches that focus on pairwise interactions, considering higher order interactions that can assess redundant and synergistic effects. In doing so, we follow the seminal ideas introduced by Tononi, Sporns, and Edelman (Tononi, et al., 1994), which postulate that higher brain functions might depend on the co-existence of integration and segregation. Indeed, while the latter enables brain areas to perform specialized tasks independently of each other, the former serves to integrate brain areas in order to perform goal-directed tasks. A key insight put forward in (Tononi, et al., 1994) is that segregation and integration can coexist, and that this coexistence is measurable by assessing the high-order interactions of neural elements via approaches such as the one used in this study.

In the context of aging, it has been shown that the balanced segregation-integration might breakdown as inter-network connectivity augments in older individuals, thereby compromising segregation. However, this increase is not associated with improved 
performance, indicating that the limited segregation is related to neuronal dysfunction, possibly due to the weaker inhibitory activity in older adults (King, et al., 2018).

It is worth noting that the increased redundancy detected in aging cannot be cast in terms of pairwise interactions. Redundancy not only implies that two areas share information but also, that the shared information is the same. Notably, no pairwise network approach can discriminate this relationship. Therefore, more redundancy in the brain does not merely imply higher connectivity but rather, that the same information is present in more than two places at the same time (the number depending on order of redundancy), which in turn suggests a loss of specificity and potentially the sub-optimal utilisation of resources.

Analyzing the O-Information, we identified different patterns of redundancy and synergy associated with increasing interaction $\operatorname{order}(n)$. While synergy exhibited a bell-shaped curve, redundancy increased linearly with $n$. It is also important to emphasize that the two metrics $\mathrm{R}$ and $\mathrm{S}$ are built in a similar way, and therefore the different scaling behavior when increasing $\mathrm{n}$ might be due to the differences in the nature of interactions separately for R and S. Although further work is needed to fully clarify the tendency when systematically increasing $n$ in $S$ and $R$, it would be possible that each brain region, organized differently to process information differently, had a well-defined internal synergetic scale, that somehow might depend on multiple factors, such as morphological, circuit, or cellular characteristics, or the region size. However, this seems not to happen with $\mathrm{R}$, that scales linearly with $\mathrm{n}$. Therefore, for very high orders, $\mathrm{R}$ would dominate over S. In a different context, using the paradigmatic Ising model for high-order interactions and emerging behaviour, recent work (Marinazzo et al., 2019) has shown that synergy peaks in the disordered phase, while redundancy does it at the critical temperature, thus showing fundamental emerging macroscopic behaviour between $\mathrm{S}$ and $\mathrm{R}$ when pooling together different high-order interactions. Additionally, previous work (Rosas et al., 2018) has shown that a balance of synergy at small and redundancy at large scales is associated to complex, emergent behaviors, such those present in certain elementary cellular automata. Deepening our understanding of these issues, their underlying enabling mechanisms, and their implications, is an important avenue for future research. 
The current study is based on the following methodological limitations. First, that the accurate quantification of redundancy in large-scale brain networks remains unresolved (Lizier, et al., 2018), although by using the O-information measure to reflect the balance of synergy-redundancy, we propose a different metric as a proxy to estimate synergy and redundancy. Second, different brain parcellations can be used to explore higher order functional interactions in the brain, although a finer spatial resolution compromises the calculation of all of the $n$-plets when assessing the O-Information when the combinations become very large. Third, the fact that the 0 -Information values themselves suggest a predominance of redundancy might be heavily influenced by the nature of the fMRI data, and different results might be obtained if other parameters were used (e.g. EEG or MEG). Nevertheless, the increase in O-Information is relevant as it is clear evidence of a change in the differentiation-integration balance towards more redundant arrangements. Finally, in the context of aging, we have shown major variations of $\mathrm{R}$ and $\mathrm{S}$ across brain structures occurring in the old population by taking into consideration exclusively fMRI. How much of these variations due to age in $\mathrm{R}$ and $\mathrm{S}$ might be originated by age-variations in the structural connectivity matrix is an unexplored territory, that needs to be addressed in future work.

In summary, the framework presented here provides a novel insight into the aging brain by assessing high order functional interdependencies among brain regions. The data reveal the role of redundancy in prefrontal and motor cortices in older participants, which affects basic functions like working memory, or executive and motor functions. We believe that this approach may help to provide a better understanding of some brain disorders from an informational perspective, providing distinctive patterns of higher order functional behavior or "info-markers", which may offer fundamental insights into the healthy and diseased human brain. The code to compute the metrics used in this article has been made publicly available at www.github.com/brincolab/High-Order-interactions.

\section{Acknowledgments}

The authors thank Karine Bertin for her valuable comments on our work. M.G. was partially sup- ported by CONICYT-PFCHA/ Doctorado Nacional/ 2019-21190577. R.C. acknowledges financial support from Fondecyt Iniciación 2018 Proyecto 11181072. P.M. 
was funded by the Wellcome Trust (grant no. 210920/Z/18/Z). F.R. acknowledges the support of the Ad Astra Chandaria Foundation. P.O. is funded by Fondecyt Regular grant 1181076 and ANID-Basal Project FB0008. S.P.S was supported by the FWO Research Foundation Flanders (G089818N), the Excellence of Science funding competition (EOS; 30446199) and the KU Leuven Special Research Fund (grant C16/15/070). J.M.C acknowledges financial support from Ikerbasque (The Basque Foundation for Science) and from the Ministerio Economia, Industria y Competitividad (Spain) and FEDER (grant DPI2016-79874- R), as well as from the Department of Economic Development and Infrastructures of the Basque Country (Elkartek Program,KK-2018/00032 and KK2018/00090). The Centro Interdisciplinario de Neurociencia de Valparaíso (CINV) is a Millennium Institute supported by Grant ICN09-022 (ICM-ANID).

Preprint posted on bioRxiv. Date posted: 18/03/2020 id: 2020.03.17.995886

\section{Authorship Confirmation Statement}

Marilyn Gatica: study conceptualization, methodology, performed the analyses, produced the figures and drafted the manuscript. Rodrigo Cofré: study conceptualization, methodology, performed the analyses, supervised the information metrics and drafted the manuscript. Pedro A.M. Mediano: study conceptualization, methodology, supervised the information metrics and drafted the manuscript. Fernando E. Rosas: study conceptualization, methodology, supervised the information metrics and drafted the manuscript. Patricio Orio: study conceptualization and methodology. Ibai Diez: preprocessed the images. S.P. Swinnen: recruited the participants and drafted the manuscript. Jesus M. Cortes: study conceptualization, data curation, methodology, supervised the information metrics and drafted the manuscript. All the authors participated in the writing of the article and agreed on the submission of the final version. Author(s') Disclosure Statement(s)

The authors declare no conflict of interest 


\section{Bibliography}

Abbas, K., Shenk, T., Poole, V., Breedlove, E., Leverenz, L., Nauman, E., Talavage, T., and Robinson, M. (2015). Alteration of default mode network in high school football athletes due to repetitive subconcussive mild traumatic brain injury: a resting-state functional magnetic resonance imaging study. Brain Connect, 5:91-101.

Andrews-Hanna, J. R., Snyder, A. Z., Vincent, J. L., Lustig, C., Head, D., Raichle, M., and Buckner, R. L. (2007). Disruption of Large-Scale Brain Systems in Advanced Aging. Neuron, 56(5):924-935.

Battiston, F., Cencetti, G., Iacopinic, I., Latora, V., Lucas, M., Patania, A., Young, J., and Petri, G. (2020). Networks beyond pairwise interactions: structure and dynamics". Physics Reports, 874: 1-92.

Beckmann, C., DeLuca, M., Devlin, J., and Smith, S. (2005). Investigations into resting-state connectivity using independent component analysis. Philos Trans R Soc Lond B Biol Sci, 360:1001-1013.

Benjamini, Y. and Hochberg, Y. (1995). Controlling the false discovery rate: A practical and powerful approach to multiple testing. Journal of the Royal Statistical Society: Series B (Methodological), 57(1):289-300.

Betzel, R. F., Byrge, L., He, Y., Goñi, J., Zuo, X. N., and Sporns, O. (2014). Changes in structural and functional connectivity among resting-state networks across the human lifespan. Neurolmage, 102(P2):345-357.

Buckner, R., Andrews-Hanna, J., and Schacter, D. (2008). The brain's default network: anatomy, function, and relevance to disease. Ann N Y Acad Sci, 1124:1-38.

Camino-Pontes, B., Diez, I., Jimenez-Marin, A., Rasero, J., Erramuzpe, A., Bonifazi, P., Stramaglia, S., Swinnen, S., and Cortes, J. (2018). Interaction Information Along Lifespan of the Resting Brain Dynamics Reveals a Major Redundant Role of the Default Mode Network. Entropy, 20(10):742. 
Chan, M. Y., Park, D. C., Savalia, N. K., Petersen, S. E., and Wig, G. S. (2014). Decreased segregation of brain systems across the healthy adult lifespan. Proceedings of the National Academy of Sciences, 111(46):E4997-E5006.

Courchesne, E., Chisum, H. J., Townsend, J., Cowles, A., Covington, J., Egaas, B., Harwood, M., Hinds, S., and Press, G. A. (2000). Normal Brain Development and Aging: Quantitative Analysis at in Vivo MR Imaging in Healthy Volunteers. Radiology, 216(3):672-682.

Damoiseaux, J. S., Beckmann, C. F., Arigita, E. J., Barkhof, F., Scheltens, P., Stam, C. J., Smith, S. M., and Rombouts, S. A. (2008). Reduced resting- state brain activity in the "default network"in normal aging. Cerebral Cortex.

Diez, I., Bonifazi, P., Escudero, I., Mateos, B., Muñoz, M. A., Stramaglia, S., and Cortes, J. M. (2015). A novel brain partition highlights the modular skeleton shared by structure and function. Scientific Reports, 5:10532.

Diez, I., Drijkoningen, D., Stramaglia, S., Bonifazi, P., Marinazzo, D., Gooijers, J., Swinnen, S. P., and Cortes, J. M. (2017). Enhanced prefrontal functional-structural networks to support postural control deficits after traumatic brain injury in a pediatric population. Netw Neurosci, 1(2):116- 142.

Erramuzpe, A., Ortega, G. J., Pastor, J., Sola, R. G., Marinazzo, D., Stramaglia, S., and Cortes, J. M. (2015). Identification of redundant and synergetic circuits in triplets of electrophysiological data. Journal of Neural Engineering, 12(6).

Friston, K. J. and Price, C. J. (2003). Degeneracy and redundancy in cognitive anatomy. Trends in Cognitive Sciences, 7(4):151-152.

Grady, C. (2008). Cognitive neuroscience of aging. Ann N Y Acad Sci, 1124:127-144.

Grady, C. (2012). The cognitive neuroscience of ageing. Nature Reviews Neuroscience, 13(7):491-505.

Han, T. S. (1978). Nonnegative entropy measures of multivariate symmetric correlations. Information and Control. 
Heuninckx, S., N Wenderoth, and Swinnen, S. (2008). Systems neuroplasticity in the aging brain: recruiting additional neural resources for successful motor performance in elderly persons. J Neurosci, 28(1):91-99.

Heuninckx, S., Wenderoth, N., F Debaere, R Peeters, and SP Swinnen (2005). Neural basis of aging: the penetration of cognition into action control. J Neurosci, 25:6787-6796.

Ince, R. A. (2017a). Measuring multivariate redundant information with pointwise common change in surprisal. Entropy, 19(7).

Ince, R. A., Giordano, B. L., Kayser, C., Rousselet, G. A., Gross, J., and Schyns, P. G. (2017b). A statistical framework for neuroimaging data analysis based on mutual information estimated via a gaussian copula. Human Brain Mapping, 38(3):1541-1573.

Jimenez-Marin, A., Rivera, D., Boado, V., Diez, I., Labayen, F., Garrido, I., Ramos-Usuga, D., Benito-Sanchez, I., Rasero, J., Cabrera-Zubizarreta, A., Gabilondo, I., Stramaglia, S., ArangoLasprilla, J., and Cortes, J. (2020). Brain connectivity and cognitive functioning in individuals six months after multiorgan failure. Neurolmage: Clinical, 25:102137.

King, B., van Ruitenbeek, P., Leunissen, I., Cuypers, K., Heise, K., Monteiro, T. S., Hermans, L., Levin, O., Albouy, G., Mantini, D., and Swinnen, S. (2018). Age-related declines in motor performance are associated with decreased segregation of large-scale resting state brain networks. Cereb Cortex, 28:4390-4402.

Knight, B. G. and Durbin, K. (2015). Aging and the effects of emotion on cognition: Implications for psychological interventions for depression and anxiety. PsyCh Journal, 4(1):11-19.

Kondratova, A. A. and Kondratov, R. V. (2012). The circadian clock and pathology of the ageing brain. Nature Reviews. Neuroscience, 13(5):325- 335.

Lizier, J. T., Bertschinger, N., Jost, J., and Wibral, M. (2018). Information decomposition of target effects from multi-source interactions: Perspectives on previous, current and future work. Entropy, 20(4). 
Luppi, A. I., Mediano, P. A., Rosas, F. E., Holland, N., Fryer, T. D., O'Brien, J. T., Rowe, J. B., Menon, D. K., Bor, D., and Stamatakis, E. A. (2020a). A synergistic core for human brain evolution and cognition. bioRxiv preprint bioRxiv: 2020.09.22.308981.

Luppi, A. I., Mediano, P. A., Rosas, F. E., Allanson, J., Pickard, J. D., Carhart-Harris, R. L., Williams, G. B., Craig, M. M., Finoia, P., Owen, A. M., Naci, L., Menon, D. K., Bor, D., and Stamatakis, E. A. (2020b). A Synergistic Workspace for Human Consciousness Revealed by Integrated Information Decomposition. bioRxiv preprint bioRxiv: 2020.11.25.398081 Marinazzo, D., Angelini, L., Pellicoro, M., and Stramaglia, S. (2019). Synergy as a warning sign of transitions: the case of the two-dimensional Ising model. arXiv preprint arXiv:1901.05405 .

Margulies, D., Vincent, J., Kelly, C., Lohmann, G., Uddin, L., Biswal, B., Villringer, A., Castellanos, F., Milham, M., and Petrides, M. (2009). Precuneus shares intrinsic functional architecture in humans and monkey. Proc Natl Acad Sci U S A, 106:20069-20074.

McGill, W. J. (1954). Multivariate information transmission. IRE Professional Group on Information Theory.

Onoda, K., Ishihara, M., and Yamaguchi, S. (2012). Decreased functional connectivity by aging is associated with cognitive decline. Journal of cognitive neuroscience.

Organization, W. H. (2018). Ageing and health. www.who.int/news-room/factsheets/detail/ageing-and-health

Pauwels, L., Maes, C., Hermans, L., and Swinnen, S. (2019). Motor inhibition efficiency in healthy aging: the role of gamma-aminobutyric acid. Neural Regen Res, 14:741-744.

Raichle, M. (2015). The brain's default mode network. Annu Rev Neurosci, 38:433-447.

Rosas, F., Mediano, P., Ugarte, M., and Jensen, H. (2018). An information- theoretic approach to self-organisation: Emergence of complex interdependencies in coupled dynamical systems. Entropy, 20(10):793. 
Rosas, F., Mediano, P. A. M., Gastpar, M., and Jensen, H. J. (2019). Quantifying High-order Interdependencies via Multivariate Extensions of the Mutual Information. Phys. Rev. E, $100,032305$.

Rueda-Delgado, L., KF Heise, A Daffertshofer, D Mantini, and SP Swinnen (2019). Agerelated differences in neural spectral power during motor learning. Neurobiol Aging, 77:44-57.

Salat, D., Buckner, R., Snyder, A., Greve, D., Desikan, R., Busa, E., Morris, J., Dale, A., and Fischl, B. (2004). Thinning of the cerebral cortex in aging. Cereb Cortex, 14:721-730.

Santos-Monteiro, T., Beets, I., Boisgontier, M., Gooijers, J., Pauwels, L., Chalavi, S., King, B., Albouy, G., and Swinnen, S. (2017). Relative cortico-subcortical shift in brain activity but preserved training-induced neural modulation in older adults during bimanual motor learning. Neurobiol Aging, 58:54-67.

Schneidman, E., Bialek, W., and Berry, M. J. (2003). Synergy, Redundancy, and Independence in Population Codes. The Journal of Neuroscience.

Schultz, A., Chhatwal, J., Hedden, T., Mormino, E., Hanseeuw, B., Sepulcre, J., Huijbers, W., LaPoint, M., Buckley, R., Johnson, K., and Sperling, R. (2017). Phases of hyperconnectivity and hypoconnectivity in the default mode and salience networks track with amyloid and tau in clinically normal individuals. J Neurosci, 37:4323-4331.

Solesio-Jofre, E., Serbruyns, L., Woolley, D. G., Mantini, D., Beets, I. A., and Swinnen, S. P. (2014). Aging effects on the resting state motor network and interlimb coordination. Human Brain Mapping.

Stramaglia, S., Angelini, L., Wu, G., Cortes, J. M., Faes, L., and Marinazzo, D. (2016). Synergetic and Redundant Information Flow Detected by Unnormalized Granger Causality: Application to Resting State fMRI. IEEE Transactions on Biomedical Engineering, 63(12):2518-2524.

Stramaglia, S., Scagliarini, T., Daniels, B. C., and Marinazzo, D. (2021). Quantifying Dynamical High-Order Interdependencies From the O-Information: An Application to Neural Spiking Dynamics. Front. Physiol. 11:595736. 
Sullivan, E. V. and Pfefferbaum, A. (2007). Neuroradiological characterization of normal adult ageing. The British Journal of Radiology, 80(special_issue_2): S99-S108.

Timme, N., Alford, W., Flecker, B., and Beggs, J. M. (2014). Synergy, redundancy, and multivariate information measures: An experimentalist's perspective. Journal of Computational Neuroscience, 36(2):119-140.

Tononi, G. and Edelman, G. M. (1998). Consciousness and complexity. Science, 282(5395):1846-1851.

Tononi, G., Sporns, O., and Edelman, G. M. (1994). A measure for brain complexity: relating functional segregation and integration in the nervous system. Proceedings of the National Academy of Sciences, 91(11):5033- 5037.

Van Putten, M. and Stam, C. (2001). Application of a neural complexity measure to multichannel eeg. Physics Letters A, 281(2-3):131-141. van Walsum, A.-M. v. C., Pijnenburg, Y., Berendse, H., van Dijk, B., Knol, D., Scheltens, P., and Stam, C. (2003). A neural complexity measure applied to meg data in alzheimer's disease. Clinical neurophysiology, 114(6):1034-1040.

Watanabe, S. (2010). Information Theoretical Analysis of Multivariate Correlation. IBM Journal of Research and Development.

West, M. J. (1993). Regionally specific loss of neurons in the aging human hippocampus. Neurobiology of Aging,14(4):287-293.

Wibral, M., Priesemann, V., Kay, J. W., Lizier, J. T., and Phillips, W. A. (2017). Partial information decomposition as a unified approach to the specification of neural goal functions. Brain and cognition, 112:25-38.

Williams, P. L. and Beer, R. D. (2010). Nonnegative decomposition of multivariate information. arXiv preprint arXiv:1004.2515. 
Figures legends
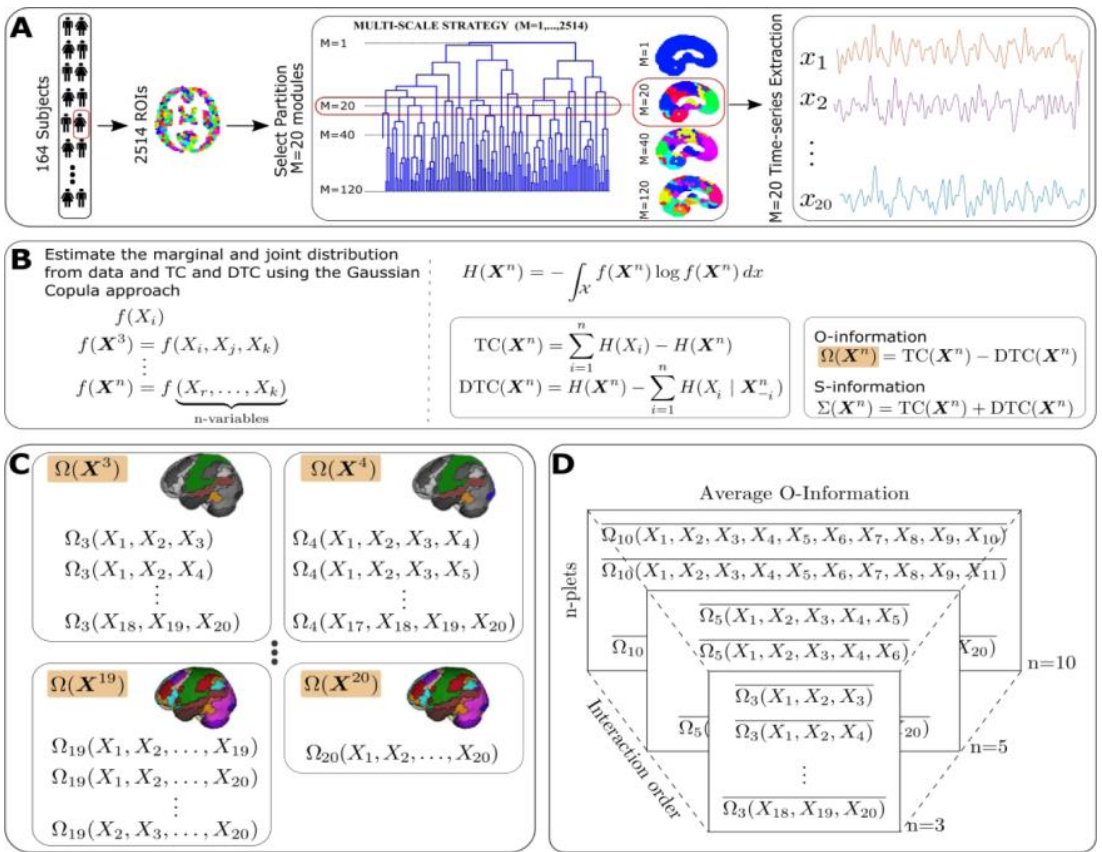

Figure 1: Methodological scheme. A: 164 subjects were included in this study, each represented by 2,514 time series of $\mathrm{fMRI}$ signals. Using The Brain Hierarchical Atlas (BHA), the original time series was grouped into $M=20$ different ones. $B$ : The marginal and joint distributions for each subject were estimated from the data using the Gaussian copula, from which the marginal and joint entropies were calculated. Next, the total correlation (TC) and dual total correlation (DTC) were obtained, from which the O- and S-Information were finally computed. C: For each subject and interaction order, we computed their OInformation and S-Information values for each n-plet, which were then used for further analyses. D: The average O-Information values of the participants were also calculated for each n-plet for group-comparisons. 

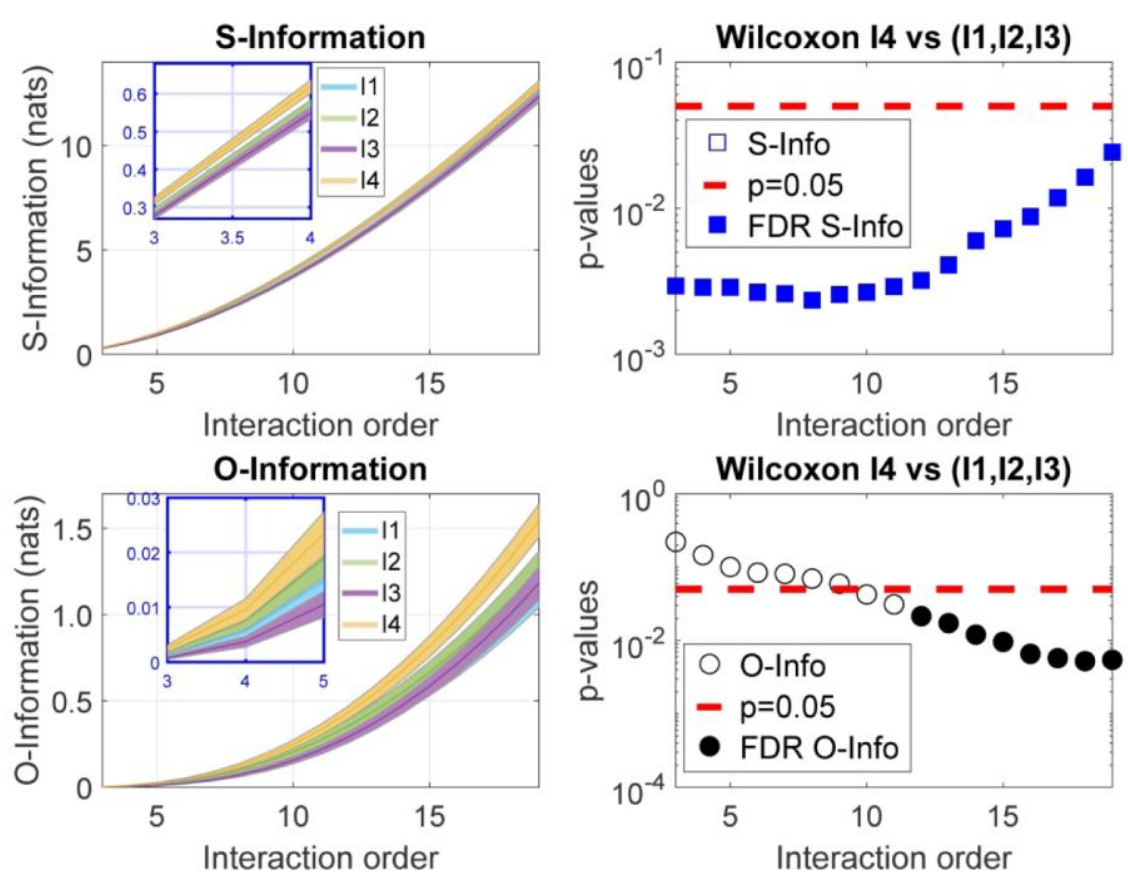

Figure 2: Strong high-order interdependencies in the aging brain. Average O-Information and S-Information average over all modules $m=1, \ldots, M$ from equations 5 and 6 for each subject,grouped as $11,12,13$, I4.Both S-Information (top panel) and O-Information (bottom panel) differentiated the higher order interdependencies in the old brains (14) relative to the younger ones (I1,I2 and 13).This is evident in the right column, where the statistical significance is represented either for a uncorrected p-value of 0.05 (red dashed line) or corrected by the FDR (represented by blue-filled squares for the S-Information and by black-filled circles for the O-Information). The interaction order is shown on the $\mathrm{x}$-axis in all the plots. 

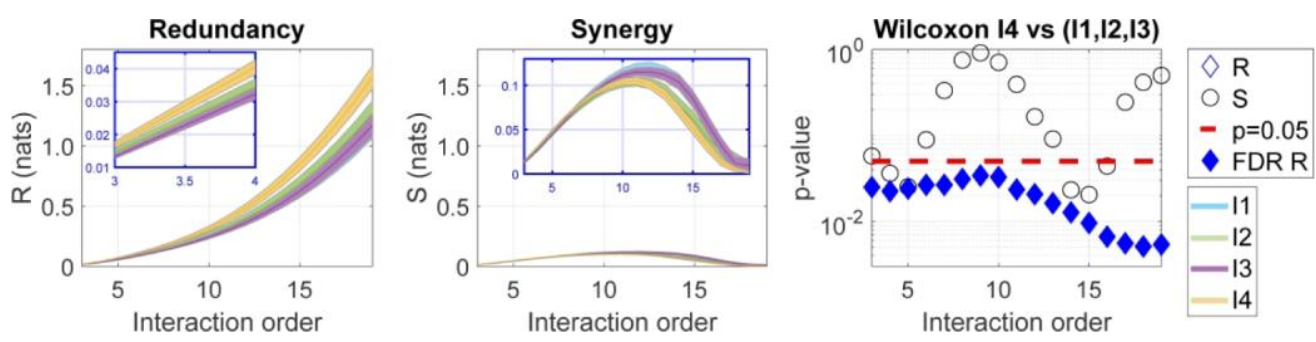

Figure 3: A significant increase in redundancy in older participants across all interaction orders. The average redundancy and synergy were obtained over modules $m=1, \ldots, M$ through the equations (10) and (11) for each subject grouped into I1, 12, 13, 14. Note that when increasing the interaction order, and independent of age, both redundancy and synergy curves have a completely different pattern (one increasing linearly and the other following a bell-shaped curve). The right panel shows that the group differences in redundancy (represented by diamonds) in older participants (14) were significantly different from those of the other groups combined $(11,12,13)$ for all interaction orders. Regarding synergy (represented by circles), none of the values survived a correction for multiple comparisons at any of the interaction orders. Both the diamonds and circles are filled when the value of redundancy or synergy survived correction for multiple comparisons. 


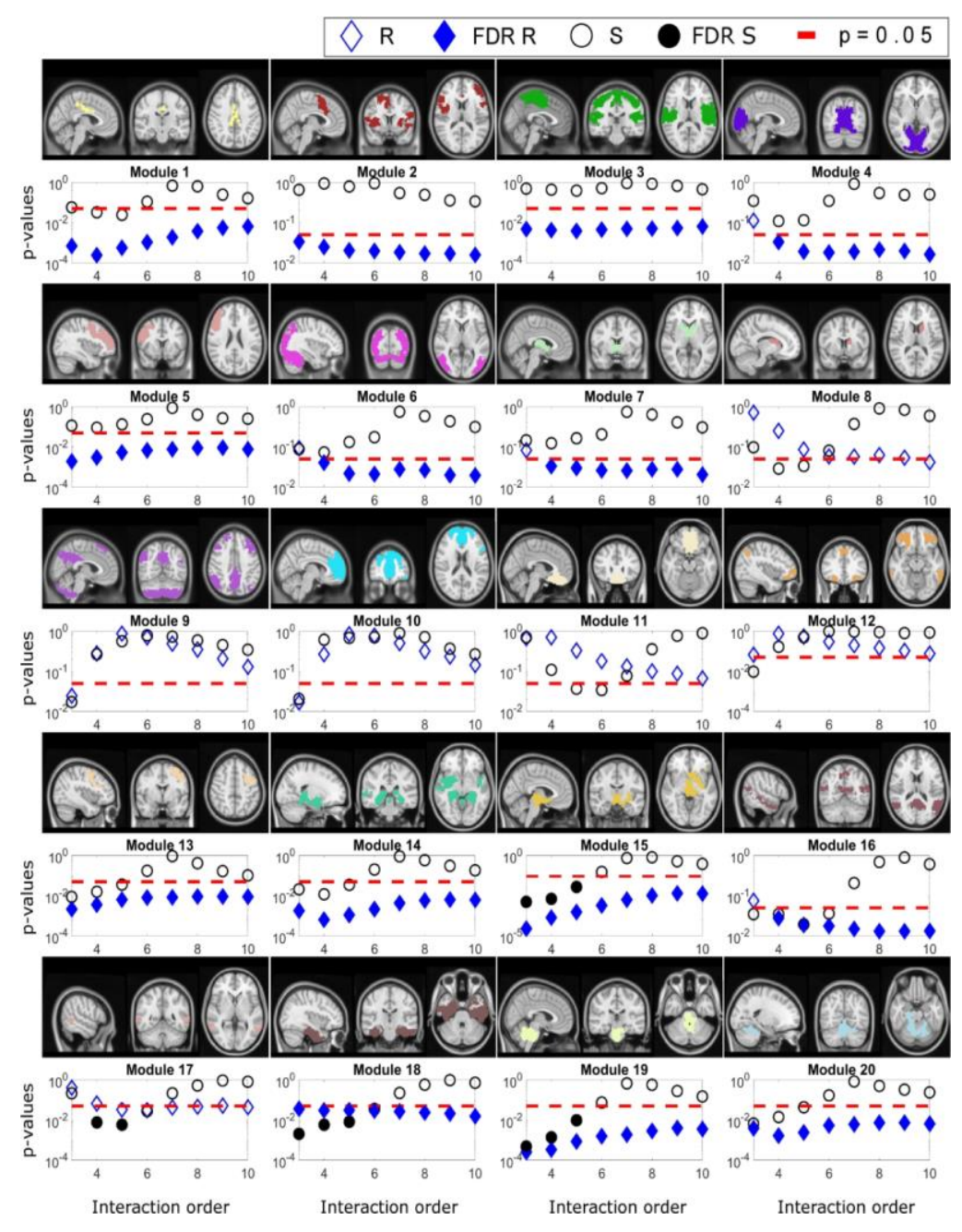

Figure 4: Higher order redundant and synergistic interdependencies across brain areas in the aging brain. Differences between 14 and the $11,12,13$ groups across the different brain modules, each one anatomically represented by three representative views from left to right, sagittal, coronal and axial. As in Fig. 3, group differences in redundancy (diamonds) and synergy (circles) are represented as a function of the interaction order. When the group differences survived correction for multiple comparisons, both the diamonds and circles are filled. In relation to redundancy, there are two "classes" of modules, those where redundancy was significantly different for any order (such as modules 1-3, 5, 13-15 and 18-20) and the remaining modules for which this did not happen. Moreover, redundancy generally differs widely between group 14 and the rest of the population across the different brain areas, yet synergy does not, for which only modules $15,17,18$ and 19 showed a few interaction orders with values surviving multiple comparisons. 

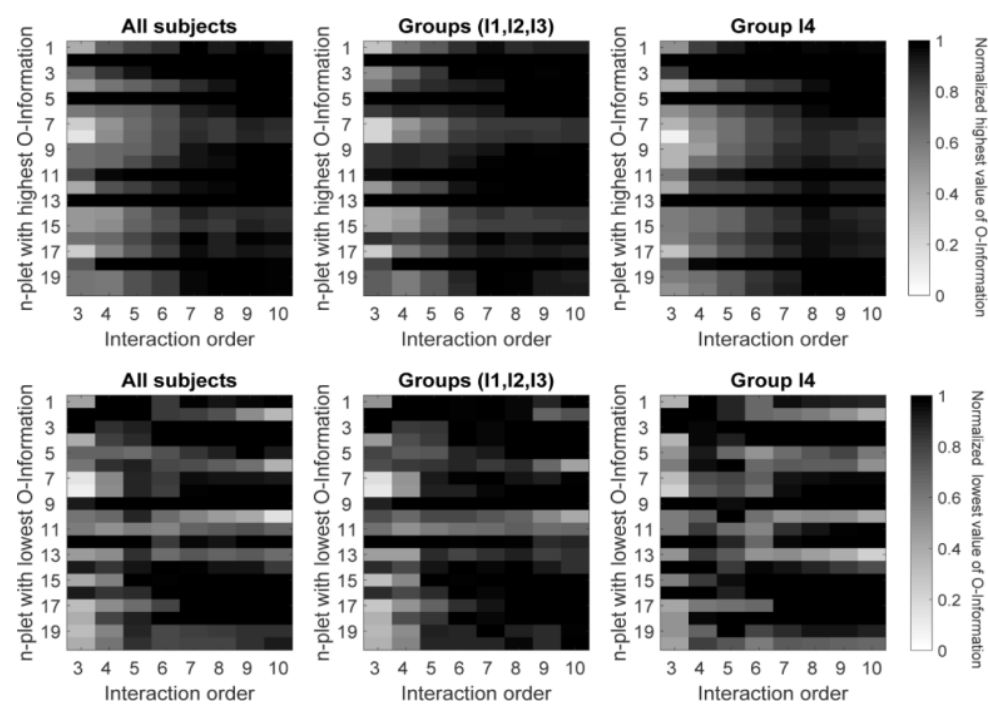

Figure 5: Identification of the redundant and synergistic cores for the different interaction orders. Top row: As a function of the interaction order $n$, we rank the averaged O-Information values of all the n-plets per group of participants, as detailed in the Methods and illustrated in Fig.1 panel D. We plotted the highest O-Information value in which each module participates for each interaction order, normalized to the n-plet with the highest O-Information averaged in different groups of participants. For each interaction order the n-plet with the highest values of O-Information are in black. The highest O-Information values measure redundancy. Bottom row: As above but plotting the lowest values of O-Information. Because all were negative, they corresponded to synergy. The two rows show similar plots for three different situations: all subjects (left); pooling I1, 12 , and 13 together (middle); and for the older group, 14 (right). 